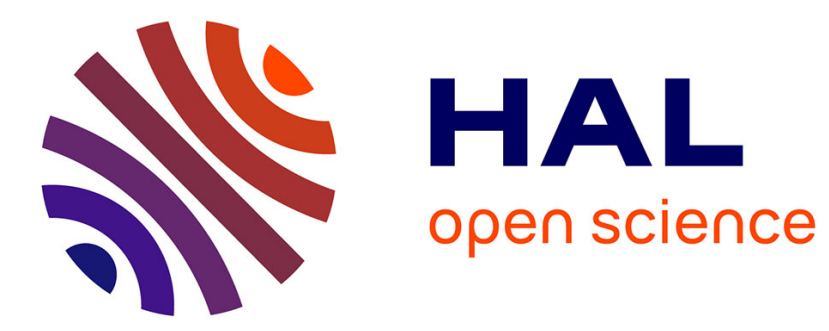

\title{
Where do I move my sensors? Emergence of a topological representation of sensors poses from the sensorimotor flow \\ Valentin Marcel, Sylvain Argentieri, Bruno Gas
}

\section{- To cite this version:}

Valentin Marcel, Sylvain Argentieri, Bruno Gas. Where do I move my sensors? Emergence of a topological representation of sensors poses from the sensorimotor flow. 2019. hal-01682639v2

\section{HAL Id: hal-01682639 \\ https://hal.science/hal-01682639v2}

Preprint submitted on 27 Mar 2019

HAL is a multi-disciplinary open access archive for the deposit and dissemination of scientific research documents, whether they are published or not. The documents may come from teaching and research institutions in France or abroad, or from public or private research centers.
L'archive ouverte pluridisciplinaire HAL, est destinée au dépôt et à la diffusion de documents scientifiques de niveau recherche, publiés ou non, émanant des établissements d'enseignement et de recherche français ou étrangers, des laboratoires publics ou privés. 


\title{
Where do I move my sensors? Emergence of a topological representation of sensors poses from the sensorimotor flow
}

\author{
Valentin Marcel, Sylvain Argentieri and Bruno Gas
}

\begin{abstract}
This paper deals with the perception of mobile robotic systems within the framework of interactive perception, and inspired by the sensorimotor contingencies (SMC) theory. These approaches state that perception arises from active exploration of an environment. In the SMC theory, it is postulated that information about the structure of space could be recovered from a quasi-uninterpreted sensorimotor flow. In a recent article, the authors have provided a mathematical framework for the construction of a sensorimotor representation of the interaction between the sensors and the body of a naive agent, provided that the sensory inputs come from the agent's own body. An extension of these results, with stimulations coming from an unknown changing environment, is proposed in this paper. More precisely it is demonstrated that, through repeated explorations of its motor configurations, the perceived sensory invariants can be exploited to build a topologically accurate internal representation of the relative poses of the agent's sensors in the physical world. Precise theoretical considerations are provided as well as an experimental framework. Finally, some examples that serve as proofs of concepts are analysed in both simulated and realistic environments.
\end{abstract}

\section{INTRODUCTION}

Space perception is a central issue in mobile robotics. Indeed, many abilities heavily depend on it as they are deeply rooted on spatial knowledge: among others, one can cite trajectory planning [1], obstacle avoidance [2], auditory and visual sensing [3], etc. Most of these works consider that space is something that exists objectively out there, and try to exploit it to model mechanical systems, localize some objects of interest, reach and catch moving targets, etc. But some other theories envisage that space has not to be a pre-established substrate per se to be able to perform the very same tasks. In the case of the sensorimotor contingencies theory (SMC) [4], [5], it is claimed that space is something that an agent may experience via the determination of sensorimotor invariants called contingencies. In other words, the discovery at first, and then the use of such contingencies, is enough to make an agent realize actions without the need of having an internal, local or global representation, analytic or not, of space. [6] has shown it unambiguously by learning a function representing any translation, whatever its origin and whatever the state of the environment, from an agent sensorimotor flow. Since then, this idea has been extended in [7] by working on the group of the two-dimensional space transformations. The underlying idea of these works is based on the notion of active compensable

V. Marcel, S. Argentieri and B. Gas are with Sorbonne Université, CNRS Institut des Systèmes Intelligents et de Robotique, ISIR, F-75005 Paris, France sensory changes proposed initially by Poincaré [8], [9]. Since then, substantial works have been published about considering action in the structuring of perception [10]-[14], some of them aiming at verifying Poincaré's idea on the notion of space, but more recently with a growing interest for robotics applications. In this vein, [15] has introduced the notion of "interactive perception" as the set of approaches in robotics concerned with the implication of action in perception.

This paper is rooted in this SMC paradigm, which has been mathematically formalized in the early 2000s [16]. This initial formalization led to the demonstration that an agent can, without any a priori, infer the so-called "dimension of space". Further works like [17] have also shown that, beyond the dimension of space, it is possible to build a motor internal representation of the positions occupied by the agent's end-effector without external knowledge about its working space. Despite the use of a curvilinear component analysis (CCA) [18] and the definition of adapted Hausdorff distances in the agent motor space, this work lacks a proper mathematical formalization and they are no clear definitions on the properties or spaces that are actually captured by the agent. As a solution, the authors have demonstrated in [19] that topological properties of the space of sensory invariants on a sensitive body can also be well captured. This work focused on the agent self-interaction with its own body, whose perception is more deeply analyzed in [20] within the SMC theory framework. Working on the agent's body was initially envisaged as a way to put the environment dependency of the representation aside. Indeed, as formalized later in this paper, the fact that the environmental state can possibly evolve along exploration has already proven to be a major theoretical difficulty [17], [21]. This paper proposes to tackle the environment dependency by generalizing the formalism initially proposed in [19]. Differently from this previous contribution, it is envisaged that the agent's end-effector is not sampling its own body anymore in such a way that all the sensory inputs are dependant to the environmental state. By defining the various environmental states as sequences, it is shown that an agent can undergo, by successive iterations, a partitioning process of its motor set. Starting from an unstructured motor set, the agent will build little by little a final motor partition which finally forms a good representation of the external working space initially unknown to the agent. As such, this refinement process is conducted along the agent's life, through an active exploration of sensory invariant in changing environments.

This paper is organized as follows. Section II is devoted 
to the mathematical formalization of the aforementioned refinement process of the agent motor set. Additionally to all the theoretical considerations, a simple example is used all along the section (and in the rest of the paper) to illustrate this process and its limits. Section III introduces some formal considerations regarding the structures of the sets obtained during the previous refinement process. These theoretical points mainly deal with topological properties which could explain how and why the refinement can allow the agent to structure its motor set into a space whose properties are similar to its external working space initially unknown. Then, all this theoretical background is exploited in Section IV to propose an experimental and probabilistic framework for building this internal representation. Simulations are conducted in Section V to carefully evaluate this framework. Finally, a conclusion ends the paper.

\section{THE REFINEMENT PROCESS : PARTITIONING OF THE MOTOR SPACE THROUGH SENSORY INVARIANTS}

\section{A. Characterization of the motor space}

1) Refinement of the motor space: Let's first consider a naive agent, be it virtual or robotic, which can interact with its environment by generating motor commands lying in the motor configuration set $\mathcal{M}$. This set can be described by latent variables parameterizing the agent actuators states (i.e. joint angles, positions, etc.), thus forming a motor state $\boldsymbol{m} \in \mathcal{M}$. The agent is also endowed with sensors rigidly placed on its own body parts. These sensors inform the agent about the environment's physical state, thus generating a sensory input $s \in \mathcal{S}$, with $\mathcal{S}$ the sensory set. Of course, the sensory input $s$ relates to the agent motor state $\boldsymbol{m}$ through the sensorimotor law $\Psi$, so that in a given environmental state $\epsilon \in \mathscr{E}$, $s=\Psi_{\boldsymbol{\epsilon}}(\boldsymbol{m})$. This relation also outlines the dependency of the sensory input $s$ to the environmental state $\epsilon$, with $\mathscr{E}$ the set of all possible environmental states.

Because of the possible redundancies in the agent kinematics, the function $\Psi_{\epsilon}($.$) is possibly non-injective. It means that,$ at $\boldsymbol{\epsilon}$, two different motor states $\boldsymbol{m}_{1}$ and $\boldsymbol{m}_{2}$ can lead to the very same sensory input $s=\Psi_{\boldsymbol{\epsilon}}\left(\boldsymbol{m}_{1}\right)=\Psi_{\boldsymbol{\epsilon}}\left(\boldsymbol{m}_{2}\right)$. As outlined in the previous authors work [19], one can then define an equivalence relation $=_{\Psi_{\epsilon}}$, such that

$$
\boldsymbol{m}_{1}=\Psi_{\boldsymbol{\epsilon}} \boldsymbol{m}_{2} \Leftrightarrow \Psi_{\boldsymbol{\epsilon}}\left(\boldsymbol{m}_{1}\right)=\Psi_{\boldsymbol{\epsilon}}\left(\boldsymbol{m}_{2}\right) .
$$

Thus, one can regroup all the motor states leading to the same sensory state in their equivalence class $[\boldsymbol{m}]_{=_{\Psi_{\epsilon}}}=[\boldsymbol{m}]_{\epsilon}=$ $\left\{\boldsymbol{r} \in \mathcal{M} \mid \boldsymbol{r}={ }_{\Psi_{\boldsymbol{\epsilon}}} \boldsymbol{m}\right\}$. It is well known that the set of all equivalence classes forms a partition ${ }^{1}$ of the set on which the equivalence relation is defined. In other words, every element in $\mathcal{M}$ is included in one and only one equivalence class $[\boldsymbol{m}]_{\boldsymbol{\epsilon}}$. This partition is called the quotient set $\mathcal{M} / \boldsymbol{\epsilon}=\left\{[\boldsymbol{m}]_{\boldsymbol{\epsilon}} \mid \boldsymbol{m} \in\right.$ $\mathcal{M}$. Additionally, it forms a refinement of the trivial partition $\{\mathcal{M}\}$ of $\mathcal{M}$, i.e. every element in the trivial equivalence class $\mathcal{M}$ is composed of subsets of $\mathcal{M} / \epsilon$.

Consider now that the environmental state has switched from $\epsilon$ to $\epsilon^{\prime}$. Then the agent has access to a new equivalence

\footnotetext{
${ }^{1}$ A partition of a set $X$ is a set of non-empty, pairwise disjoints, subsets whose union forms the set $X$ itself.
}

relation $=\Psi_{\epsilon^{\prime}}$, that leads to a new motor set partition $\mathcal{M} / \epsilon^{\prime}$. It should be highlighted that for any $\boldsymbol{m}_{1}, \boldsymbol{m}_{2} \in \mathcal{M}, \boldsymbol{m}_{1}={ }_{\Psi_{\epsilon}}$ $\boldsymbol{m}_{2} \leftrightarrow \boldsymbol{m}_{1}=_{\Psi_{\epsilon^{\prime}}} \boldsymbol{m}_{2}$ and $\boldsymbol{m}_{1} \neq_{\Psi_{\boldsymbol{\epsilon}}} \boldsymbol{m}_{2} \leftrightarrow \boldsymbol{m}_{1} \neq \Psi_{\epsilon_{\epsilon^{\prime}}} \boldsymbol{m}_{2}$. It clearly means that the two partitions $\mathcal{M} / \epsilon$ and $\mathcal{M} / \epsilon^{\prime}$ can't be directly compared, as they are both representing one specific motor partition dedicated to a given environmental state. However, one can define a new multi-environment equivalence relation $=_{\left(\epsilon, \epsilon^{\prime}\right)}$ as

$$
\boldsymbol{m}_{1}={ }_{\Psi_{\left(\epsilon, \epsilon^{\prime}\right)}} \boldsymbol{m}_{2} \Leftrightarrow\left(\begin{array}{cc}
\boldsymbol{m}_{1}={ }_{\Psi_{\boldsymbol{\epsilon}}} & \boldsymbol{m}_{2} \\
\text { and } & \\
\boldsymbol{m}_{1}={ }_{\Psi_{\epsilon^{\prime}}} & \boldsymbol{m}_{2}
\end{array}\right) .
$$

According to its definition, this equivalence relation leads to equivalent classes $[\boldsymbol{m}]_{\left(\boldsymbol{\epsilon}, \boldsymbol{\epsilon}^{\prime}\right)}=[\boldsymbol{m}]_{\boldsymbol{\epsilon}} \cap[\boldsymbol{m}]_{\boldsymbol{\epsilon}^{\prime}}$ verifying $[\boldsymbol{m}]_{\left(\boldsymbol{\epsilon}, \boldsymbol{\epsilon}^{\prime}\right)} \subseteq[\boldsymbol{m}]_{\boldsymbol{\epsilon}}$ and $[\boldsymbol{m}]_{\left(\boldsymbol{\epsilon}, \boldsymbol{\epsilon}^{\prime}\right)} \subseteq[\boldsymbol{m}]_{\boldsymbol{\epsilon}^{\prime}}$, i.e. the quotient set $\mathcal{M} /{ }_{\left(\epsilon, \epsilon^{\prime}\right)}$ is a refinement of both quotient sets $\mathcal{M} / \epsilon_{\epsilon}$ and $\mathcal{M} /{ }_{\epsilon^{\prime}}$. Note that this multi-environment equivalence relation $=\Psi_{\left(\epsilon, \epsilon^{\prime}\right)}$ does not depend on the order of $\epsilon$ and $\epsilon^{\prime}$. Consequently, the tuple $\left(\boldsymbol{\epsilon}, \boldsymbol{\epsilon}^{\prime}\right)$ can be written as a subset $E=\left\{\boldsymbol{\epsilon}, \boldsymbol{\epsilon}^{\prime}\right\}$ of $\mathscr{E}$. Based on the idea that intersecting partitions obtained on multiple environments gives a finer partition, one can then define the generic multi-environment equivalence relation $={ }_{\Psi_{E}}$, for any subset $E \subseteq \mathscr{E}$, as

$$
\begin{aligned}
\boldsymbol{m}_{1}=\Psi_{E} \boldsymbol{m}_{2} & \Leftrightarrow \forall \boldsymbol{\epsilon} \in E, \Psi_{\boldsymbol{\epsilon}}\left(\boldsymbol{m}_{1}\right)=\Psi_{\boldsymbol{\epsilon}}\left(\boldsymbol{m}_{2}\right), \\
& \Leftrightarrow \Psi_{E}\left(\boldsymbol{m}_{1}\right)=\Psi_{E}\left(\boldsymbol{m}_{2}\right),
\end{aligned}
$$

where the function $\Psi_{E}$ maps each motor configuration to its respective sensory sequence acquired along a succession of environmental states $\epsilon \in E$, i.e.

$$
\begin{aligned}
\Psi_{E}: & \mathcal{M} \\
\mathcal{M} & \mapsto \prod_{\boldsymbol{\epsilon} \in E} \mathcal{S} \\
\mathcal{M} & \left.\mapsto \Psi_{\boldsymbol{\epsilon}}(\boldsymbol{m})\right)_{\boldsymbol{\epsilon} \in E}
\end{aligned}
$$

with $\prod$ the Cartesian product of sets. The corresponding set of equivalence classes is the quotient set noted $\mathcal{M} / E$.Adding new environmental states to a subset $E$ always give a more refined partition. Then, considering the extreme case where $E=\mathscr{E}$, it is clear that $\mathcal{M} / \mathscr{E}$ is the finest partition the agent can have access to. Indeed it is made of equivalence classes which can never be further fragmented because there are no new experiences possible. In that sense, its equivalent classes constitutes the so-called finest sensitive points which are closely related to points in physical space, as illustrated later in $\S$ II-B3.

2) Illustrative example: All the aforementioned considerations were mainly theoretical. Let's now illustrate these points by using a very simple simulated robot agent made of one serial arm composed of two parts of identical length controlled by two revolute joints moving in a plane, see figure 1 . The end-effector of the system is endowed with a 1-pixel (punctual) camera which is only sensitive to illumination in such a manner that it can only send two values: $s=0$ if the illumination is zero and $s=1$ otherwise. The system is driven by two motor commands $\theta_{1}$ and $\theta_{2}$, which are supposed to represent directly the two joint angles, so that by convention $\theta_{1}, \theta_{2} \in\left[-\pi, \pi\left[\left(\theta_{1}=\theta_{2}=0\right.\right.\right.$ makes the arm horizontal on the right in Figure 1(a). Suppose now that the environment is 
made of a black and a white areas separated by a straight line, as depicted on top of Subfigures 1(a) and 1(b). Of course, the agent does not have access to this information and can only rely on its sensorimotor flow, i.e variations of $\theta_{1}, \theta_{2}$ and their sensory consequences. At the very beginning, the set of all motor commands $\boldsymbol{m}=\left(\theta_{1}, \theta_{2}\right) \in \mathcal{M}=\left[-\pi, \pi\left[^{2}\right.\right.$ have not been distinguished from each other so that the current finest motor partition is $\{\mathcal{M}\}$. After having explored one black and white environment, the agent is able to obtain a finer motor partition. Indeed, two equivalent classes can easily be formed by regrouping all the motor commands $\boldsymbol{m}$ giving the same sensation, namely, for an environmental state $\boldsymbol{\epsilon},\left[\boldsymbol{m}_{0}\right]_{\boldsymbol{\epsilon}}$ for $s=0$ and $\left[\boldsymbol{m}_{1}\right]_{\boldsymbol{\epsilon}}$ for $s=1$. Then, the set $\left\{\left[\boldsymbol{m}_{0}\right]_{\boldsymbol{\epsilon}},\left[\boldsymbol{m}_{1}\right]_{\boldsymbol{\epsilon}}\right\}$ forms a partition of the set $\{\mathcal{M}\}$, which can be represented as two separated points, see Figure 1(c). This partition is also colored directly in the motor set in Figure 1(a). Of course this partition is environment dependent, which is captured in the formalization with a dependency to the environmental state $\epsilon$ in the equivalence relation $=_{\Psi_{\epsilon}}$. This dependency has been discussed in many publications [16], [17], [21], [22], and no clear solutions have been proposed so far to deal with this environment variability. In these works, the environment is systematically considered static, and they often restrict their study to cases where the environment changes do not influence the sensorimotor flow (by working on the agent body, for instance, like in [19] and [23]).

However if the environmental state changes to a new state $\epsilon^{\prime}$ (corresponding to a new black and white separation of the robot working space, as shown in Figure 1(b)), then it is possible that previously inseparable motor configurations (regrouped in one equivalence class) are now generating different sensations. Considering this new environmental state $\epsilon^{\prime}$ alone, it is clear that the agent can partition its motor set into two equivalence classes $\left[\boldsymbol{m}_{0}\right]_{\boldsymbol{\epsilon}^{\prime}}$ and $\left[\boldsymbol{m}_{1}\right]_{\boldsymbol{\epsilon}^{\prime}}$, thus leading to a new motor partition shown in Figure 1(b). Remembering the previous partition, the agent can now build a finer partition for having sequentially experimented the environmental states $\boldsymbol{\epsilon}$ and $\boldsymbol{\epsilon}^{\prime}$. The resulting multi-environment partitioning can be easily deduced in this case, and is shown in Figure 1(c). In this intuitive example, the agent is now able to separate the equivalence class $\left[\boldsymbol{m}_{0}\right]_{\epsilon}$, which relates to all the motor configurations giving the same 0 sensation value for the environmental state $\epsilon$, into two new subsets that are denoted $\left[\boldsymbol{m}_{00}\right]_{E}=\left[\boldsymbol{m}_{0}\right]_{\boldsymbol{\epsilon}} \cap\left[\boldsymbol{m}_{0}\right]_{\boldsymbol{\epsilon}^{\prime}}$ and $\left[\boldsymbol{m}_{01}\right]_{E}=\left[\boldsymbol{m}_{0}\right]_{\boldsymbol{\epsilon}} \cap\left[\boldsymbol{m}_{1}\right]_{\boldsymbol{\epsilon}^{\prime}}$, with the sequence $E=\left\{\boldsymbol{\epsilon}, \boldsymbol{\epsilon}^{\prime}\right\} .\left[\boldsymbol{m}_{1}\right]_{\boldsymbol{\epsilon}}$ is also partitioned in two subsets $\left[\boldsymbol{m}_{10}\right]_{E}$ and $\left[\boldsymbol{m}_{11}\right]_{E}$. Following the colors used in Figure 1(c), one can then illustrate this refinement with the down arrows in Figure 1(d).

If the experiment is iteratively reproduced, then the multienvironment partition will again be refined, with all the equivalence classes being more and more partitioned into smaller subsets. The refined sets would then shows a monotonically growing number of points along with the number of environmental states observed. In this example, there is a possibly infinite number of environment states that the agent can interact with so that the number of points will most likely tends to infinity: all the equivalence classes can always be further partitioned with a new specific environment. However, by considering the case where the agent has interacted with all possible environmental states (which is in reality impossible with this simple example) one obtains a case where the equivalence classes are not refinable and can be considered as points, called the finest sensitive points. But what are the finest sensitive points in the actual physical space ?

\section{B. From the motor quotient set to the sensor pose}

So far, the previous section has highlighted the only two sets the agent can be aware of: the motor configuration set $\mathcal{M}$ and the sensory set $\mathcal{S}$, where its motor states and sensory inputs respectively lie. Both sets are linked together through the sensorimotor law $\Psi$. From an external point of view, the sensory input $s \in \mathcal{S}$ is generated by rigid sensors whose spatial state in the world can be entirely described by their pose in the world: $\boldsymbol{x} \in \mathcal{X}$, with $\mathcal{X}$ the sensors pose set. Let's focus on this new set and highlight the links between $\mathcal{X}, \mathcal{M}$ and $\mathcal{S}$.

1) Definition of the sensor pose set: It is well known in robotics that the forward kinematics function $f$, which accounts for the relative movements allowed at each joint and is dependent on the geometry of the robot, is a function linking the motor state $\boldsymbol{m}$ to the corresponding sensors pose $\boldsymbol{x}$, usually in Euclidean space, so that $\boldsymbol{x}=f(\boldsymbol{m})$. In general, the pose $\boldsymbol{x}$-which is a parameterization of the sensors spatial state in the physical world- refers to the sensors positions and their orientations relatively to the frame of the agent's body. The sensors poses are externally defined thus not directly accessible by the agent. The sensory input is thus linked to the spatial state of the sensors through the forward sensory function $\phi_{\boldsymbol{\epsilon}}$, so that $s=\phi_{\boldsymbol{\epsilon}}(\boldsymbol{x})$. In the end, the sensorimotor law $\Psi_{\epsilon}$ can be written as the composition $\Psi_{\epsilon}=\phi_{\epsilon} \circ f$, which is summarized by the diagram

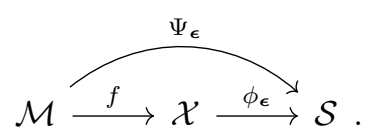

Introducing $\mathcal{X}$ is a convenient way to understand how the motor refinement, outlined in $\oint$ II-A1, is related to space. Following the same ideas, one can define an equivalence relation for two poses. At first, one can note that the two functions $f$ and $\phi_{\epsilon}$ can be both considered non-injective. This means that two different motor configurations can lead to the same sensors pose (i.e. the non-injectivity of $f$ captures the agent kinematics redundancy) and in a specific environment state $\epsilon$ two different sensors poses can lead to the same sensory state (i.e. the non-injectivity of $\phi_{\epsilon}$ captures the environmental redundancies but also the sensors possible symmetries). In the vein of Equations (1), then, for any $\epsilon \in \mathscr{E}$, one can again define an equivalence relation $={ }_{\phi_{\epsilon}}$ for two poses with

$$
\boldsymbol{x}_{1}={ }_{\phi_{\boldsymbol{\epsilon}}} \boldsymbol{x}_{2} \Leftrightarrow \phi_{\boldsymbol{\epsilon}}\left(\boldsymbol{x}_{1}\right)=\phi_{\boldsymbol{\epsilon}}\left(\boldsymbol{x}_{2}\right) .
$$

Thus, one can regroup all the sensor poses leading to the same sensory state in their equivalence class

$$
[\boldsymbol{x}]_{\boldsymbol{\epsilon}}=\left\{\boldsymbol{r} \in \mathcal{X} ; \boldsymbol{r}={ }_{\phi_{\boldsymbol{\epsilon}}} \boldsymbol{x}\right\} .
$$

Then, the quotient set $\mathcal{X} / \boldsymbol{\epsilon}=\left\{[\boldsymbol{x}]_{\boldsymbol{\epsilon}} ; \boldsymbol{x} \in \mathcal{X}\right\}$ forms a refinement of the trivial partition $\{\mathcal{X}\}$. By generalization over 


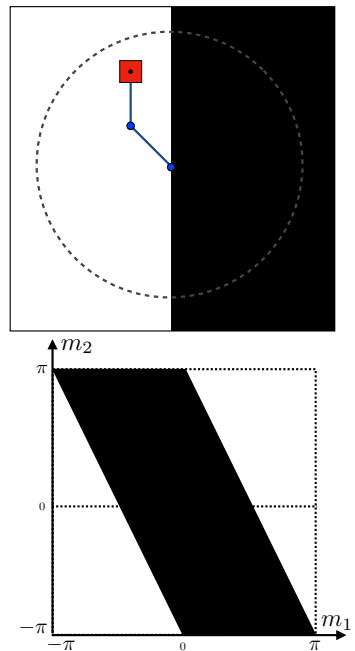

(a) Environment $\epsilon$.

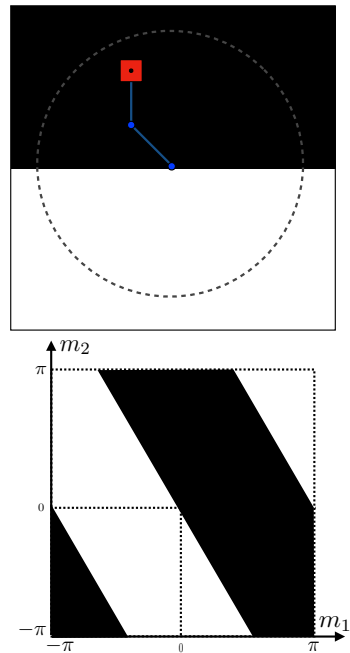

(b) Environment $\epsilon^{\prime}$.

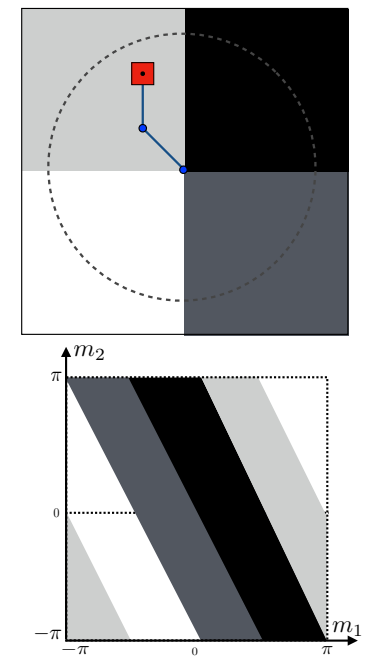

(c) Sequence $\left(\boldsymbol{\epsilon}, \epsilon^{\prime}\right)$.

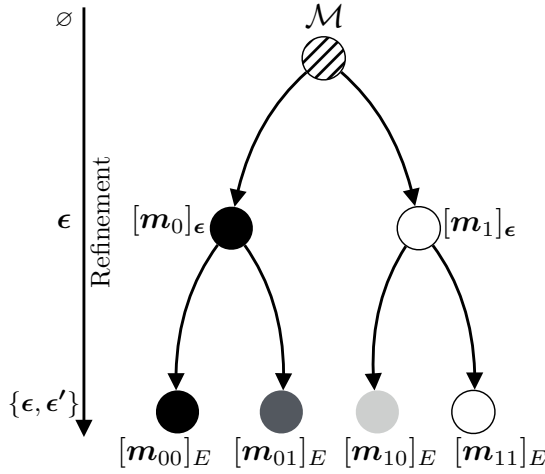

(d) Motor set refinement.

Fig. 1. Illustration of the refinement process. (Top) The 2D serial agent with 2 degrees of freedom and the 1-pixel camera (red) and its working space (circle). (Bottom) Sensorimotor clusters represented in the motor configuration set $\mathcal{M}$. (a) The agent is in an environment made of black and white areas parameterized by its state $\boldsymbol{\epsilon}$. The agent is only able to cluster its motor set in two partitions, i.e. two equivalence classes $\left[\boldsymbol{m}_{0}\right]_{\boldsymbol{\epsilon}}$ and $\left[\boldsymbol{m}_{1}\right]_{\boldsymbol{\epsilon}}$ respectively related to the only two sensations available to the agent : $s=0$ (black area) and $s=1$ (white area). (b) The same applies for the new environment configuration $\epsilon^{\prime}$. (c) In fact, the agent has experienced the environment sequence $\left(\boldsymbol{\epsilon}, \boldsymbol{\epsilon}^{\prime}\right)$, leading to the 4 sequences represented by different gray levels: black area is for sequence (black\&black), dark gray is for sequence ( black\&white), light gray is for sequence (white\&black) and white for sequence (white\&white). (d) Then, the initial two equivalence classes $\left(\left[\boldsymbol{m}_{0}\right]_{\boldsymbol{\epsilon}}\right.$ and $\left.\left[\boldsymbol{m}_{1}\right]_{\boldsymbol{\epsilon}}\right)$ built when experiencing the environmental state $\boldsymbol{\epsilon}$ can be partitioned again in 4 subsets after experiencing a new state $\epsilon^{\prime}$.

the environmental state $\epsilon \in E$, one can then define the multienvironment equivalence relation $={ }_{\phi_{E}}$ for any subset $E \subseteq \mathscr{E}$ defined along

$$
\boldsymbol{x}_{1}={ }_{\phi_{E}} \boldsymbol{x}_{2} \Leftrightarrow \phi_{\boldsymbol{\epsilon}}\left(\boldsymbol{x}_{1}\right)=\phi_{\boldsymbol{\epsilon}}\left(\boldsymbol{x}_{2}\right), \forall \boldsymbol{\epsilon} \in E,
$$

where the function $\phi_{E}$ maps each sensor pose to its respective sensory sequence acquired along the successive environmental states $\epsilon \in E$, i.e.

$$
\begin{aligned}
\phi_{E}: \quad \mathcal{X} & \rightarrow \prod_{\boldsymbol{\epsilon} \in E} \mathcal{S} \\
\boldsymbol{x} & \mapsto\left(\phi_{\boldsymbol{\epsilon}}(\boldsymbol{x})\right)_{\boldsymbol{\epsilon} \in E}
\end{aligned}
$$

The equivalence relation $={ }_{\phi_{E}}$ can be understood as: two poses are said equivalent after having seen all environments in $E \subseteq$ $\mathscr{E}$ if the sensory inputs they have produced are equal for all environmental states in $E$. These poses can then be regrouped in a equivalence class

$$
[\boldsymbol{x}]_{E}=\left\{\boldsymbol{r} \in \mathcal{X} ; \boldsymbol{r}={ }_{\phi_{E}} \boldsymbol{x}\right\} .
$$

The set of all equivalence classes is the quotient set $\mathcal{X} / E$. Like before, the extreme case where $E=\mathscr{E}$ is of particular interest. Indeed, $\mathcal{X} / \mathscr{E}$ is made of equivalence classes which can not be further fragmented into subsets, thus defining the finest sensitive pose space. It is interesting to see that $\mathcal{X}$ is in fact a refinement of $\mathcal{X} / \mathscr{E}$. This highlights the fact that there might exists some subsets of positions and orientations in the sensor's working space that can never be distinguished from sensory inputs. Then, from an internal point of view using the sensorimotor flow, the agent will never be capable to separate those ambiguous subsets and is unable to represent the whole pose set $\mathcal{X}$.
2) Interlink between $\mathcal{M} /{ }_{E}$ and $\mathcal{X} /_{E}$ : So far, two quotient sets have been introduced: (i) the motor quotient set $\mathcal{M} / E$ which can be built directly from the sensorimotor flow and by interaction between the agent and its environment, and (ii) the sensitive working space $\mathcal{X} / E$ which captures sensor poses that have not been distinguished from sensory inputs. However, it is clear that, by definition, the two equivalence relations $={ }_{\Psi_{E}}$ and $={ }_{\phi_{E}}$ both give the same number of equivalence classes in their respective sets. It then immediately follows that for any subset $E \subseteq \mathscr{E}, \mathcal{X} / E$ and $\mathcal{M} /{ }_{E}$ are equinumerous. Then, from an external point of view, the link between all the sets so far defined can be subsumed by the following diagram

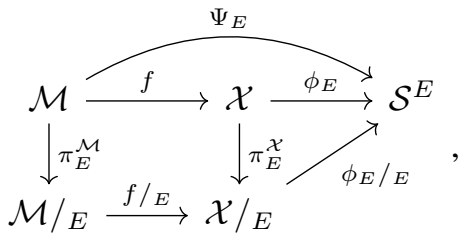

where $f / E$ represents the unique bijective map mapping together equivalence classes from $\mathcal{M} / E$ to $\mathcal{X} / E \cdot \pi_{E}^{\mathcal{M}}$ and $\pi_{E}^{\mathcal{X}}$ both represents the canonical projections from points to equivalence classes. Consequently, the agent can exploit $\mathcal{M} / E$ as an internal representation of $\mathcal{X} / E$. Furthermore, letting $E=\mathscr{E}$ means then that the agent has experienced all the possible environmental states, i.e. each point in $\mathcal{X} / \mathscr{E}$ is equivalently represented by a finest sensitive point in the internal representation $\mathcal{M} / \mathscr{E}$. These considerations are illustrated in the following subsection.

3) Illustrative example (cont'd): Let's come back to the previous illustrative example, where a two-DOF robot arm explores a black and white environment. In this simple case:

- the environmental state $\epsilon$ can be fully described by a 
straight line delimiting the working space in two areas together with a binary value indicating which one is black;

- the agent's motor configuration set $\mathcal{M}$ is made of the set of the two joint angles $\theta_{1}, \theta_{2}$ so that $\mathcal{M}=$ $\left\{\left(\theta_{1}, \theta_{2}\right) ; \theta_{1}, \theta_{2} \in[-\pi, \pi[\}\right.$;

- the forward kinematics function $f$ gives the punctual endeffector position $(x, y) \in \mathcal{X} \subset \mathbb{R}^{2}$ as a function of $\theta_{1}, \theta_{2}$ and $L$, the length of the two arm parts, with $(x, y)=$ $\left(L\left(\cos \theta_{1}+\cos \left(\theta_{1}+\theta_{2}\right)\right), L\left(\sin \theta_{1}+\sin \left(\theta_{1}+\theta_{2}\right)\right)\right)$;

- the pointwise sensor, placed on $(x, y)$, delivers a sensa$\operatorname{tion}^{2} \boldsymbol{s}=\phi_{\boldsymbol{\epsilon}}(x, y) \in \mathcal{S}=\{0,1\}$.

The agent is there endowed with a pointwise sensor, so a pose $\boldsymbol{x}$ in $\mathcal{X}$ is nothing else but a point in a 2D Euclidean space. Since two distinct points in the $2 D$ Euclidean plan can always been separated by a straight line, equivalently for two distinct poses in $\mathcal{X}$, there always exists an environmental state $\epsilon \in \mathscr{E}$ for which the corresponding sensations are distinct. Then, for a given pose $\boldsymbol{x}$, the equivalent class $[\boldsymbol{x}]_{\mathscr{E}}$ regrouping all the poses that always give the same sensations for all $\epsilon \in \mathscr{E}$ is just the singleton $[\boldsymbol{x}]_{\mathscr{E}}=\{\boldsymbol{x}\}$. This means that the finest partition $\mathcal{X} / \mathscr{E}$ of the working space is the set of points in $\mathcal{X}$, i.e. $\mathcal{X} / \mathscr{E}=\{\{(x, y)\},(x, y) \in \mathcal{X}\}$.

Following the same ideas, it is clear that the equivalence classes in the motor configurations set $\mathcal{M}$ are the set of motor configurations leading to a same and unique pose in the working space through the forward kinematics function $f$. Consequently, the finest partition $\mathcal{M} / \mathscr{E}$ is made of equivalence classes $[\boldsymbol{m}]_{\mathscr{E}}$ individually corresponding to one equivalence class $[\boldsymbol{x}]_{\mathscr{E}}=[f(\boldsymbol{m})]_{\mathscr{E}}$. However the finest sensitive points in $\mathcal{M} / \mathscr{E}$ have a unique corresponding point in $\mathcal{X} / \mathscr{E}$, which have been shown to represent points in the working space. Then, without any knowledge on the forward kinematics function $f$ -and through a refinement strategy- the agent can build the set $\mathcal{M} / \mathscr{E}$ which captures kinematics redundancies and constitutes a very good candidate for representing the working space.

All these considerations are represented in Figure 2. The working space is represented in the middle, where each pose can be reached by the agent from one or multiple motor configurations due to the kinematics redundancy. For instance, the pose $\boldsymbol{x}_{1}$ (resp. $\boldsymbol{x}_{3}$ ) can be reached by the 2 different motor configurations $\boldsymbol{m}_{1}$ and $\boldsymbol{m}_{2}$ (resp. $\boldsymbol{m}_{3}$ and $\boldsymbol{m}_{4}$ ) in $\mathcal{M}$. The same applies for the pose $\boldsymbol{x}_{5}$ located at the limit of the working space, which can be obtained with a unique motor configuration $\boldsymbol{m}_{5}$. Another particular case is the pose $\boldsymbol{x}_{6}$ obtained when the sensor is exactly in the center of the working space, which can be reached with all motor configurations $\boldsymbol{m}=\left(\theta_{1}, \theta_{2}\right)$ such that $\theta_{2}=-\pi$, thus building the set $\mathscr{M}_{6} \in \mathcal{M}$. As explained above, each of these poses is linked to an equivalent class in $\mathcal{M} / \mathscr{E}$ once the agent has experienced all the possible environmental states $\epsilon$ in $\mathscr{E}$. For instance, the two motor configurations $\boldsymbol{m}_{1}$ and $\boldsymbol{m}_{2}$ (resp. $\boldsymbol{m}_{3}$ and $\boldsymbol{m}_{4}$ ) can be regrouped in the equivalent class $\left[\boldsymbol{m}_{1}\right]_{\mathscr{E}}=\left\{\boldsymbol{m}_{1}, \boldsymbol{m}_{2}\right\}$ (resp. $\left.\left[\boldsymbol{m}_{3}\right]_{\mathscr{E}}=\left\{\boldsymbol{m}_{3}, \boldsymbol{m}_{4}\right\}\right)$ in $\mathcal{M} / \mathscr{E}$. Then, one can see on this illustration that each undividable equivalent class obtained on

\footnotetext{
${ }^{2}$ In the particular case where the sensor is placed exactly on the straight line splitting the working space in two areas, it is arbitrary chosen that $s=0$.
}

the finest partition $\mathcal{M} / \mathscr{E}$ forms a finest sensitive point, each of them being associated to a unique pose in the working space, i.e. a point in the 2D Euclidean space. Thus, the agent knows for instance that any motor configuration selected in $\mathscr{M}_{6}$ will correspond to a sensitive pose point $\left[\boldsymbol{m}_{6}\right]_{\mathscr{E}}$ (with $\boldsymbol{m}_{6} \in \mathscr{M}_{6}$ ) and so to a unique pose in the finest sensitive pose space. In that sense, one can qualitatively understand that the finest refinement represents the kernels of the forward kinematics function.

From the mathematical formalism previously proposed, one seems to have definitely concluded on the way an agent can refine its motor configurations from the sensory sequences generated during the environment exploration. Indeed, from Equation (3), it is sufficient to have one difference of sensations in the sensory sequence associated to two motor configurations to make them belong to two different equivalence classes. But what would actually happen if the sensory sequence agrees on an infinite number of observations, while being different for a single one? It is clear that in this specific case, the probability of having two different sensations in this sensory sequence may be equal to zero: then, this property can not be measured by the agent, it is not observable.

\section{On the observability of the finest refinement}

1) Formalism: It is assumed that is possible to define the probability, possibly zero, to the event: a certain environmental states occur. The space of environmental states being possibly continuous, one can further assume the existence of a probability measure $P$ on the set of environmental states. This measure gives the probability $P(E)$ to sample an environmental state from the subset $E \subseteq \mathscr{E}$. Any subset $E$ of $\mathscr{E}$ that have a probability measure $P(E)>0$ is then called measurable, meaning that there is nonzero probability to observe at least one element of it. Therefore, one could derive a new definition of the finest sensory equivalence classes on the basis on this probability measure. Indeed, if one consider a non-measurable subset of $E$, there is a probability of 0 to obtain a environmental state $\epsilon \in E$ that distinguishes two different poses : in other terms, the sensory distinguishability of such poses is unobservable by the agent. Then, one can extend the concept of finest sensitive points to finest observable sensitive points. From the probability measure $P$ on the set of environmental states $\mathscr{E}$, lets $={ }_{P}$ be the new equivalence relation such that, for any pair $\boldsymbol{x}_{i}, \boldsymbol{x}_{j} \in \mathcal{X}$ of poses,

$$
\boldsymbol{x}_{i}={ }_{P} \boldsymbol{x}_{j} \Leftrightarrow P\left(\left\{\epsilon \in \mathscr{E} ; \boldsymbol{x}_{i} \neq_{\phi_{\epsilon}} \boldsymbol{x}_{j}\right\}\right)=0 .
$$

This equivalence relation can be interpreted as: in order for two poses to be in the same equivalence class, it suffices that the probability to observe en environmental state that separates them is null. Let's now denote by $\mathcal{X} /_{P}=\left\{[\boldsymbol{x}]_{P} ; x \in \mathcal{X}\right\}$ the finest observable sensitive pose set made of the equivalence classes given by the equivalence relation ${ }_{P}$. From an external point of view, $\mathcal{X} / P$ is the finest set of points that can be observed from the sensorimotor flow; it is thus all that can be represented by the agent. Obviously, one can also define 

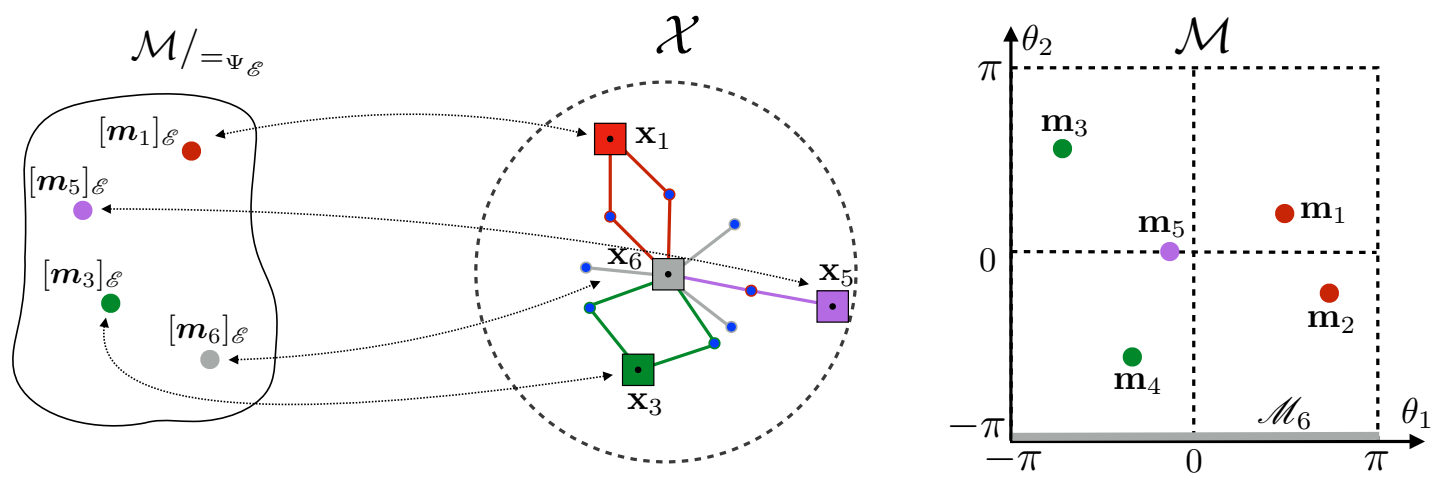

Fig. 2. Illustration of the link between $\mathcal{M}, \mathcal{X}$ and $\mathcal{M} / \mathscr{E}$ for the simple example used in the paper. (Left) Sensorimotor representative set, i.e. the finest partition of the motor set. (Middle) Pose set, with the punctual camera represented as a square. (Right) Motor configuration set. In the end, and for the finest motor representation, each sensorimotor refinement point $\left[\boldsymbol{m}_{i}\right]_{\mathscr{E}}$ in $\mathcal{M} / \mathscr{E}$ represents one and only one point in the agent working space.

the equivalence relation on the set of motor configurations to obtain the agent internal representation

$$
\boldsymbol{m}_{i}={ }_{P} \boldsymbol{m}_{j} \Leftrightarrow P\left(\left\{\epsilon \in \mathscr{E} ; \boldsymbol{m}_{i} \neq_{\Psi_{\epsilon}} \boldsymbol{m}_{j}\right\}\right)=0 .
$$

And define $\mathcal{M} / P=\left\{[\boldsymbol{m}]_{P} ; \boldsymbol{m} \in \mathcal{M}\right\}$ as the internal representation. With the same considerations than in $\S \mathrm{II}-\mathrm{B} 2$, $\mathcal{M} /{ }_{P}$ has the same number of points than in $\mathcal{X} / P$. All these properties can be subsumed in the following diagram

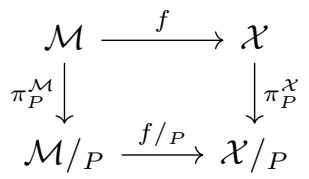

where $f / P$ denotes the bijection that maps the equivalence classes together, i.e. $f /_{P}:[\boldsymbol{m}]_{P} \rightarrow[f(\boldsymbol{m})]_{P}$ for any $\boldsymbol{m} \in$ $\mathcal{M} . \pi_{P}^{\mathcal{M}}$ and $\pi_{P}^{\mathcal{X}}$ both represents the two canonical projections from points to equivalence classes.

2) Illustrative example (cont'd): Let's apply these new considerations to the previous example. Considering Figure 2, if one takes two separated poses in the working space and choose at random a straight line intersecting with the working space, the probability to have a sensory difference between them is different from 0 . Indeed, if the distribution of straight lines intersecting with the working space is uniform, there is non null probability to separate two distinct points with this straight line. Therefore, the finest sensitive pose set $\mathcal{X} / \mathscr{E}$ and the observable sensitive pose set $\mathcal{X} / P$ are identical. Moroever in this case, the probability measure $P$ qualitatively gives a notion of distance between poses, the farther they are, the higher is the probability to distinguish them because the subset of environmental states that separates them is bigger.

The next section investigates how the probability measure $P$ gives a structural information on the internal representation and how it is related to the structure of the physical space.

\section{INTRODUCING STRUCTURAL CONSIDERATIONS INTO THE MATHEMATICAL FORMALISM}

So far, it has only been shown that a refinement process allows the agent to obtain a set of points $\mathcal{M} /{ }_{P}$ in bijection with the set of observable sensitive poses in the set $\mathcal{X} / P$. Until now, the representation is correct if the internal representation captures the finest observable sensitive points. However, in order to be exploitable, the agent should also be able to represent the continuity of movements in the physical space. Yet, there are no guarantee that close points in $\mathcal{X} / P$ are represented by close finest observable sensitive points in $\mathcal{M} / P$. These considerations are carefully addressed in the following.

\section{A. Sensorimotor structures on the quotient spaces}

From a mathematical point of view, $\mathcal{X} / P$ and $\mathcal{M} / P$ have been described as sets, and not spaces. Transforming these two sets into spaces requires the introduction of some additional structure. In fact, it is possible to derive an intrinsic dissimilarity measure between the points of these sets as they can be linked together by the probability measure $P$ on the set $\mathscr{E}$. Indeed, let's consider any pair of poses $\boldsymbol{x}_{i}, \boldsymbol{x}_{j} \in \mathcal{X}$. Then from the probability measure $P$, one can derive the probability $p\left(\boldsymbol{x}_{i}, \boldsymbol{x}_{j}\right)$ for the event "experience an environment generating two different sensations at $\boldsymbol{x}_{i}$ and $\boldsymbol{x}_{j}$ ". This can be formalized by splitting up the set of environmental states $\mathscr{E}$ in two complementary subsets $E_{i j}$ and $E_{i j}^{c}$, with $E_{i j}$ the set of environmental states such that $\forall \boldsymbol{\epsilon} \in E_{i j}, \phi_{\boldsymbol{\epsilon}}\left(\boldsymbol{x}_{i}\right) \neq$ $\phi_{\boldsymbol{\epsilon}}\left(\boldsymbol{x}_{j}\right)$. Then, using $P$, the aforementioned probability is given by $p\left(\boldsymbol{x}_{i}, \boldsymbol{x}_{j}\right)=P\left(E_{i j}\right)$. Consequently, the probability on the observable sensitive pose set $\mathcal{X} / P$ to "experience an environment generating two different sensations at two different equivalent classes $\left[\boldsymbol{x}_{i}\right]_{P}$ and $\left[\boldsymbol{x}_{j}\right]_{P}$ " is by definition $p\left(\left[\boldsymbol{x}_{i}\right]_{P},\left[\boldsymbol{x}_{j}\right]_{P}\right)=p\left(\boldsymbol{x}_{i}, \boldsymbol{x}_{j}\right)$. Importantly, it can be shown that $p$ forms a metric in the set $\mathcal{X} /{ }_{P}$, thus turning it into a metric space.

Equivalently, one can define the probability $\sigma$ on pairs of motor configurations $\boldsymbol{m}_{i}, \boldsymbol{m}_{j} \in \mathcal{M}$ to observe an environmental state that generate different sensations with $\sigma\left(\boldsymbol{m}_{i}, \boldsymbol{m}_{j}\right)=p\left(f\left(\boldsymbol{m}_{i}\right), f\left(\boldsymbol{m}_{j}\right)\right)=P\left(E_{i j}\right)$, and its extension on the internal representation $\mathcal{M} / P$ by $\sigma\left(\left[\boldsymbol{m}_{i}\right]_{P},\left[\boldsymbol{m}_{j}\right]_{P}\right)=$ $p\left(\left[f\left(\boldsymbol{m}_{i}\right)\right]_{P},\left[f\left(\boldsymbol{m}_{j}\right)\right]_{P}\right) . \sigma$ is also a metric on $\mathcal{M} / P$ and will be called in the following the sensory dissimilarity. Therefore, it appears the agent can actually builds a metric space $\left(\mathcal{M} /{ }_{P}, \sigma\right)$ by exploiting the refinement process highlighted in the previous section. Moreover, the bijective map

$$
\left(\mathcal{M} /{ }_{P}, \sigma\right) \stackrel{f /_{P}}{\longrightarrow}(\mathcal{X} / P, p)
$$


can be shown to be a bijective isometry: both spaces are thus linked together by a distance-preserving transformation.

One have to keep in mind that the metric structures induced by the probability measure $P$ is inherited from the statistics of sensorimotor invariants. Consequently, they are empirical because built directly from the observable sensorimotor experience, i.e. from the comparison of sensations along the agent life. These empirical structures, as defined previously, are not arbitrary but are an intrinsic property of the sensorimotor experience of the agent. Based on previous considerations, the space $(\mathcal{M} / P, \sigma)$ is metrically equivalent to the space $\left(\mathcal{X} /{ }_{P}, p\right)$, however does this empirical structure respects the continuity of the physical world? If not, the agent should not have any interest in building $(\mathcal{M} / P, \sigma)$ as it would not constitute a good representation of the world. This specific point is addressed in the next subsection.

\section{B. Natural structure: towards a good representation}

Before dealing with the notion of "good representation of the world", one needs to introduce natural structures in the considered spaces. Here, "natural" refers to the structures which are directly induced by the continuity of the physical world in which the agent is embedded. Indeed, the displacements of the agent sensors are hypothesized as being continuous in time and space. Therefore one can endow respectively the motor set $\mathcal{M}$ and pose set $\mathcal{X}$ with topological structures $\tau_{\mathcal{M}}$ and $\tau_{\mathcal{X}}$ that both guarantee the continuity of the forward kinematics function $f($.$) , with f:\left(\mathcal{M}, \tau_{\mathcal{M}}\right) \rightarrow\left(\mathcal{X}, \tau_{\mathcal{X}}\right)$.

So now it appears that the quotient pose set $\mathcal{X} / P$ can be endowed with two different topological structures:

- the one induced by the quotient of $\left(\mathcal{X}, \tau_{\mathcal{X}}\right)$ (i.e. the quotient topology), which captures its natural structure;

- the one induced by the metric $p$, which is empirically obtained by the agent through its sensorimotor experience.

Since one wish the empirical structure to respect the continuity of the physical world, both topological structures must be equivalent. This can be shown to be valid under the following two hypotheses:

(H1) The probability $p$ is a continuous property of the physical space, or equivalently $p$ is a continuous map from $\mathcal{X} \times \mathcal{X}$ with the product topology to $\mathbb{R}_{>0}$.

(H2) The agent's motor configuration space $\left(\mathcal{M}, \tau_{\mathcal{M}}\right)$ is compact.

Under both hypotheses it can be proven that the empirical and natural structures on the quotient set $\mathcal{X} / P$ are topologically equivalent. Additionally, since relation (14) states that $\mathcal{M} / P$ and $\mathcal{X} / P$ with their empirical structures are also topologically equivalent, then the quotient motor space $\mathcal{M} / P$ with the empirical structure is topologically equivalent with the space $\mathcal{X} / P$ endowed with the natural structure. Thus $\left(\mathcal{M} /{ }_{P}, \sigma\right)$ can be considered to be a good representation of $\mathcal{X} / P$ endowed with a structure capturing the physical continuity of space.

Now that we have stated the hypotheses under which the agent might be interested in building $\mathcal{M} /{ }_{P}$, let's focus in the next section on how to perform the refinement process from an experimental point of view.

\section{AN EXPERIMENTAL FRAMEWORK FOR THE REFINEMENT PROCESS}

The notion of refinement, together with considerations on the possible structures inherited from the agent's actions and statistics of its sensorimotor invariants, have been introduced in the two previous section. Yet, all these points were mainly theoretical: no considerations on the computational process the agent should undergo in order to obtain a correct representation have been underlined. However, the refinement is a process that will be conducted during the agent life, i.e. along time. This section is devoted to the introduction of such experimental considerations that should allow to formalize (i) what structures can actually be represented and how, and (ii) how an outside viewer can experimentally assess if this internal representation is correct. These two points are addressed in the next two subsections.

\section{A. Introducing an experimental point of view on the refinement process}

1) Experimental setup: To begin with, let's consider that the agent is totally naive and only has access to its uninterpreted sensorimotor flow. From a known home state $\boldsymbol{m}_{0}$, the agent performs a naive babbling through a set of $N$ randomly generated -but repeatable- actions, and then goes back to its home state $\boldsymbol{m}_{0}$. Through this exploration, the agent obtains a number of $N$ reads of its sensorimotor flow $\left(\boldsymbol{s}_{i}, \boldsymbol{m}_{i}\right)$, with $i=1, \ldots, N$. Let's call $M=\left\{\boldsymbol{m}_{i}\right\}_{i}$ the motor exploration set corresponding to the motor inputs. Under the hypothesis of repeatability of actions, the agent can repeat the exploration of the set $M$, each repetition being parameterized by an integer $k$. Thus, at repetition $k$ and motor configuration $\boldsymbol{m}_{i}$, the agent sensory input is $\boldsymbol{s}_{i}[k]$.

At the end of each repetition, the agent can compare sensations between pairs $(i, j)$ of all motor configurations $\left(\boldsymbol{m}_{i}, \boldsymbol{m}_{j}\right)$ in the exploration set $M$. For repetition $n$, one can define the dissimilarity matrix $\mathbf{D}[n]$ of size $N \times N$ between all pairs of motor configurations, whose elements $D_{i j}[n]$ are computed along

$D_{i j}[n]=\frac{1}{n} \sum_{k=1}^{n} \delta_{i j}[k]$, with $\delta_{i j}[k]=\left\{\begin{array}{l}0 \text { if } \boldsymbol{s}_{i}[k]=\boldsymbol{s}_{j}[k], \\ 1 \text { otherwise. }\end{array}\right.$

The elements $D_{i j}[n]$ of the dissimilarity matrix $\mathbf{D}[n]$ represent the probability for the two motor configurations $\boldsymbol{m}_{i}$ and $\boldsymbol{m}_{j}$ of being separated by a sensory input, during the sample of $n$ repetitions. Thus, it can be envisaged as an estimatorof the sensory dissimilarity $\sigma\left(\boldsymbol{m}_{i}, \boldsymbol{m}_{j}\right)$ defined in $\S$ III-A.

2) Experimental internal representation: It is clear from Eq. (15) that if at each repetition $k$ corresponds a fixed environmental state $\epsilon_{k}$, then the zeros in $D_{i j}[n]$ actually represent the equivalence classes mentioned in the formalization in $\S \mathrm{II}$ for a explored set $M$ and a sequence of $n$ environmental states. Thus, at the beginning of the agent life, i.e. when $n=0, \mathbf{D}[0]$ can be initialized as a null matrix: all motor 
configurations have not been distinguished from each other (see the initial partition in the top of Figure 1(d)). Then, as the number of repetitions increases, the agent can notice that during some repetitions the two sensations $\boldsymbol{s}_{i}[n]$ and $\boldsymbol{s}_{j}[n]$ might differ. Then, for pairs wher $D_{i j}[n]>0$ : the agent motor configurations set $M$ can be partitioned as illustrated in the graph of Figure 1(d).

Now, it is overambitious to state that as $n$ tends to infinity the dissimilarity $D_{i j}$ converges in probability towards $\sigma\left(\boldsymbol{m}_{i}, \boldsymbol{m}_{j}\right)$. Some theoretical requirements are needed on both the probability measure $P$ and the stochastic process describing the succession of environmental states. Moreover the case where the environment is fixed during a repetition is purely theoretical. Dealing with a real-life scenario requires to take into account a continuously changing environment. These changes might cause distortions in the dissimilarity $D_{i j}$ w.r.t. to the theoretical dissimilarity $\sigma\left(\boldsymbol{m}_{i}, \boldsymbol{m}_{j}\right)$. However, because the agent does not explore all its motor configurations space, it cannot represent its entire pose quotient space with its natural structure. The dissimilarity can rather be interpreted as an estimation of the metric structure of the space where the agent sensors have moved. Considerations about the link between the structures arising from the agent refinement process in a realistic environment are carefully developed in the following.

\section{B. Evaluation of the representation}

So far, it has been proven that, under some hypotheses, the agent is theoretically able to capture topological properties of the quotient pose space. In the experimental case, it is not possible to assess the convergence of the refinement process at a topological level because the explored points are discrete, and the discrete topology is trivial. The evaluation based on topological continuity cannot be performed and should be replaced by the evaluation of preservation of local structures in the form of "small" neighborhoods. Points that are close in one space should correspond to close points in the other space. Indeed it has been shown that the internal representation is a finite metric space which is equivalent to a fully connected, undirected, weighted graph where points are nodes, and edges are weighted by the distance between the two linked points. However the natural structure on the quotient pose space does not contain any closeness property. For the purpose of evaluation, let's arbitrarily introduce a stronger structure in the represented space.

1) Evaluation metric and local structure in the represented space: Let's assume that the represented space, e.g. the quotient pose space $\mathcal{X} /{ }_{P}$, is endowed with a metric $\rho$, called the evaluation metric, known to an external viewer and compatible with the natural topological structure in the quotient pose space $\mathcal{X} / P$. This metric could be used in traditional applications to derive cost functions for tasks such as path planning. In the experimental case, when the agent explores the set of motor configurations $M$, it runs through the discrete set $X=f(M)$ of poses. After taking the quotient by regrouping points that are theoretically not distinguishable from a sensory point of view, we obtain the subspace $X /{ }_{P} \subset \mathcal{X} /{ }_{P}$ for which $\rho$ is also metric. Therefore, the space to be represented by the agent is the discrete set $X / P$ with the distance matrix $\mathbf{R}$ whose elements $R_{i j}$ corresponds to the distances between elements in $X /_{P}: \rho\left(\left[\mathbf{x}_{i}\right]_{P},\left[\mathbf{x}_{j}\right]_{P}\right)$. The discrete metric space $\left(X /{ }_{P}, \mathbf{R}\right)$ can also be represented as a weighted graph.

2) Evaluation criterion: From one side we have the internal representation $(M, \mathbf{D})$ which evolves with the time of exploration, and on the other side the represented space $\left(X /{ }_{P}, \mathbf{R}\right)$. We propose two useful evaluation criterion to evaluate the structure similarity between these two spaces. The first criterion will guaranty that the agent has distinguished all points that can theoretically be distinguished. The second criterion, gives an evaluation on the conservation of the local structure between the represented and the representative graphs.

a) The refinement criterion $C_{1}$ : this criterion is the percentage of pairs of configurations $(i, j)$ that have not yet been distinguished yet by a sensory dissimilarity, but are distinguished in the quotient pose space, i.e. the number of pairs such that $D_{i j}=0$ but $R_{i j}>0 . C_{1}$ can be computed along

$$
C_{1}=\frac{\mid\left\{(i, j) ; D_{i j}=0 \text { and } R_{i j} \neq 0\right\} \mid}{N^{2}} .
$$

The finest refinement is then obtained when $C_{1}=0$, meaning that all distinct points in the quotient pose space are distinct in the internal representation.

b) Local structure similarity criterion $C_{2}$ : it is a measure of how well small neighborhoods are preserved between the dissimilarity $\mathbf{D}$ and the evaluation metric $\mathbf{R}$. This criterion is inspired by the adjusted Locally Continuous MetaCriteria (LCMC) used in Local Multi-Dimensional Scaling (LMDS) [24]. The adjusted LCMC is mainly used in the context of nonlinear multidimensional reduction. It evaluates both the preservation of continuity and the trustfulness of an embedding from measures of dissimilarity on a dataset to a low dimensional Euclidean space. It does so by comparing the neighborhood structures in both spaces. The choice of adjusted LC meta-criteria is also justified by the fact that it is a nonmetric criterion, as it uses ranks in the dissimilarities and not metric information, and so is invariant to monotonous scaling of the dissimilarities and the evaluation metric.

The adjusted LCMC is computed as follows. Let's $\mathcal{N}_{K}^{\mathrm{D}}(i)=\left\{j_{1}, \cdots, j_{K}\right\}$ be the K-Nearest Neighbors (K-NNs) of configuration $i$ with regard to dissimilarity matrix $\mathbf{D}$, and $\mathcal{N}_{K}^{\mathbf{R}}(i)=\left\{k_{1}, \cdots, k_{K}\right\}$ the K-NNs with regard to the evaluation metric $\mathbf{R}$. Then the neighborhood similarity for point $i$ is simply the cardinality of their common K-NNs:

$$
N_{K}(i)=\left|\left(\mathcal{N}_{K}^{\mathbf{D}}(i) \cap \mathcal{N}_{K}^{\mathbf{R}}(i)\right)\right| .
$$

The adjusted LCMC is given in its global form by a normalized and adjusted average over all points by

$$
Q(K)=\frac{1}{K N} \sum_{i=1}^{N} N_{K}(i)-\frac{K}{N-1} .
$$

A value of $Q(K)$ close to 1 indicates a high similarity between all the K-NNs in both spaces. However the adjusted LCMC is a function of the number of nearest neighbors: the higher $K$, the bigger the considered neighborhoods. But the only interest of the criterion $C_{2}$ is to evaluate similarity on "local structures" 
associated to a small value of $K$. This can be achieved thanks to the approach in [25], which consists in finding the value $K_{\max }$ of $K$ which maximizes the adjusted LCMC along

$$
K_{\max }=\operatorname{argmax} Q(K) .
$$

Then, criterion $C_{2}$ is computed as the average below $K_{\max }$ of $Q$ along

$$
C_{2}=\frac{1}{K_{\max }} \sum_{K=1}^{K_{\max }} Q(K) .
$$

The quantity $C_{2}$ assesses the similarity of local structures, e.g. the preservation of neighbors insides the $K_{\max }$-NNs. $K_{\max }$ represents the scale that correspond to "local" considerations. The value $C_{2}=1$ indicates that all the neighborhoods of all points of size inferior and equal to $K_{\max }$ are perfectly preserved. The value $C 2=0$ can be interpreted as a random permutation of the points.

3) $2 D$ visualization: Additionally to the two criterion introduced previously, it is also possible to project the dissimilarity matrix $\mathbf{D}$ to a low-dimensional Euclidean space by using multidimensional scaling (MDS). This projection can then be visualized to assess qualitatively the resemblance between the projected internal representation and the actual quotient pose space. However, this visualization can not replace the two quantitative criterion $C_{1}$ and $C_{2}$ in the general case, since the quotient pose space cannot always be embedded into a $2 D$ or $3 D$ Euclidean space without big distortions. The algorithm used for the projection is Isomap [26]. Given a value $K$, Isomap performs Classical MDS using geodesic distances on the K-NN graph of the dissimilarity matrix D. The visualization being not a criterion per se, any nonmetric multidimensional scaling algorithm that preserves local structures such as Local-MDS, SOM, LLE, tSNE or Curvilinear Component Analysis could have been selected. The choice of Isomap is based on its simplicity and the fact that the neighborhood scale $K$ is already available from the computation of the local similarity criterion $K_{\max }$ in Eq. (19).

The process for building the internal representation has now been formalized. Two criterion have been introduced to evaluate if the space $(M, \mathbf{D})$ is a good representative of local structures in $(X / P, \mathbf{R})$. The next section shows the results of the implementation of the refinement process for a simulated agent in different environments.

\section{Simulated RESUlTS AND DisCUSSION}

This section aims at providing a proof of concept on how a naive agent can build an internal representation of its working space in an unknown environment with an uninterpreted sensorimotor flow. Therefore, the simulated agent will be tested on different environments. The detailed simulation setup is described in a first subsection. Then, quantitative and qualitative evaluations of the obtained representations for two different scenarios of increasing complexity are proposed.

\section{A. Simulation setup}

The agent used in the simulations is the simple 2 degrees of freedom agent introduced in $\S$ II-A2. For all scenarios, the agent's motor exploration set $M$ is chosen in the following way. First, as written in the illustrative example in II-B3, recall that the motor configurations of the agent is represented with the tuple $\boldsymbol{m}=\left(\theta_{1}, \theta_{2}\right)$ where $\theta_{1}$ and $\theta_{2}$ are the two joint angles of the serial arm. The agent starts from a home position $\boldsymbol{m}_{0}=(0,0)$, the sensor being at the far right of the working space. It then generates random actions simulated by an addition of two random angles sampled from a uniform probability distribution in $[0,2 \pi]$ after which it goes back to its home position $\boldsymbol{m}_{0}$. After the $i$-th action, the agent receives the motor configuration $\boldsymbol{m}_{i}$. Without further exploration, the explored pose space would be a set of random points inside a $2 D$ disk. In order to have a better visualization of the structures in the explored space, some motor configurations are artificially added and some removed. The obtained target space of sensor poses is then shown in figure 3(a) where different colors are highlighting these structural clues. At the end, the explored motor configuration space $M$ is composed of $N=500$ motor configurations and sensors poses for every scenario. At the end of repetition $n$, the agent computes the dissimilarity distance $\mathbf{D}[n]$ with equation (15) between all pairs of explored configurations. For the simulated environments, the space to be represented is the pose space $X /{ }_{P}=X=f(M)$, where $f$ is the forward kinematics of the agent, and the evaluation metric is the euclidean distance between the poses $R_{i j}=\rho\left(\boldsymbol{x}_{\boldsymbol{i}}, \boldsymbol{x}_{\boldsymbol{j}}\right)=\left\|\boldsymbol{x}_{i}-\boldsymbol{x}_{j}\right\|_{2}^{2}$.

\section{B. Environments description and results}

The simulated environments are separated in 2 scenarios of increasing complexity. All environments are black and white or gray-scale backgrounds on the working space of the $2 \mathrm{D}$ agent as shown in the three subfigures 3(b), 3(c) and 3(d). The first scenario is composed of 2 different environments. In this first scenario the refinement process matches with the theory: the environmental states are kept fixed during the exploration of $M$, the sensory inputs are either 0 or 1 . This corresponds to the theoretical set-up formalized in section II. The second scenario is more realistic and is composed of 3 different environments. In this second scenario the environmental states are allowed to change during the exploration as it would happen in a realistic environment, and the agent's sensory inputs can take more values than 0 or 1 .

1) Scenario 1, static environment during exploration:

a) Straight lines environment: In this first environment, the environmental states are identical to those in the illustrative example and are depicted in subfigure 3(b). Each environmental state is randomly chosen as a straight line crossing the working space separating the background in one black and one white areas. The distribution of these straight lines is taken so that they uniformly cover the working space, see method 2 of Bertand's Paradox [27]. The agent's sensory input is either 1 or 0 according to which side of the straight line the agent's sensor is in. At the end of each repetition, a new straight line is randomly chosen and the refinement process continues. This process is repeated until $n=10^{6}$ explorations of the motor exploration set.

Figure 4 plots the evolution of the refinement criterion $C_{1}$. The finest refinement is obtained after almost 1400 repetitions, 


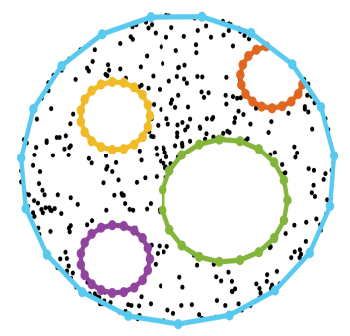

(a) Original working space with structural clues

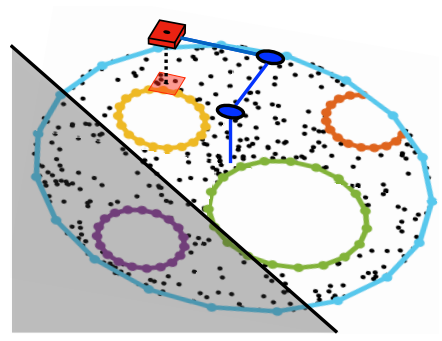

(b) (Straight lines) environment

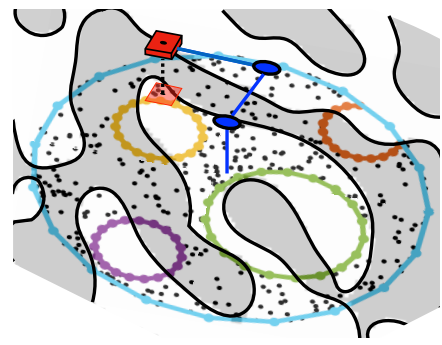

(c) (Blobs) environment

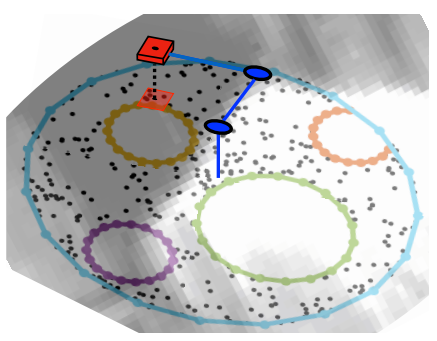

(d) (Movie) environment

Fig. 3. Far left: the $2 D$ agent's working space after exploring a motor configuration set $M$ with $N=500$ configurations. The explored sensor poses are black dots and the structural clues are linked colored dots. Right: the three different background environments.

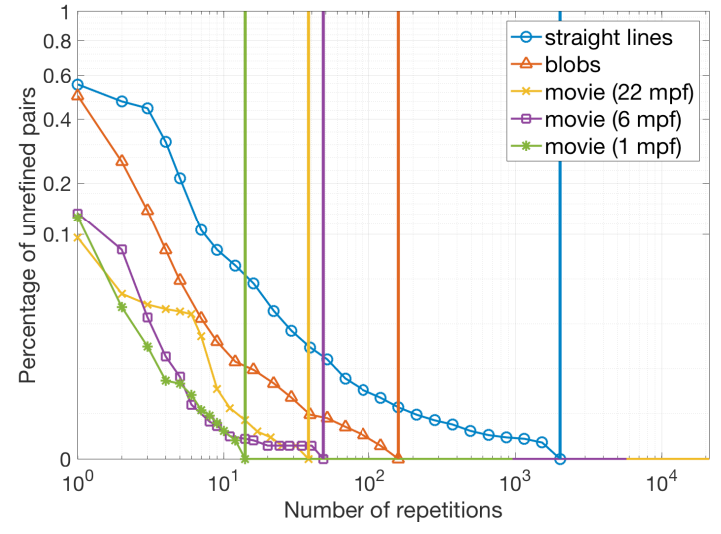

Fig. 4. Evolution of the refinement criterion $C_{1}$ for the 5 different environments. The vertical lines show at which repetition the finest refinement $\left(C_{1}=0\right)$ is obtained.

meaning that the environment is very slow at separating points in the representation. It is indeed very structured by opposition to random sensory values over the working space. In Figure 6 is plot the evolution of the local similarity criterion $C_{2}$ between the measured dissimilarity $\mathbf{D}[n]$ at repetition $n$ and the Euclidean distance $\mathbf{R}$ between the poses of the target pose space shown in subfigure 3(a). The criterion $C_{2}$ starts very low for the first exploration and then converge toward a value of $C_{2}=0.98$ which is almost a perfect match of local neighborhoods. Indeed, for this environment the statistics of sensory invariants are invariant to translations or rotations in the $2 D$ Euclidean working space [28] and in fact it can be proved that the measured dissimilarity converges in probability to the Euclidean distance between the poses, up to a constant factor.

In order to interpret the values of $C_{2}$ and $C_{1}$, let's visualize the $2 D$ projection of the obtained dissimilarity matrix along the agent life using Isomap. The results of the visualization for the different environments are shown in Figure $5^{3}$. The internal representation starts with a few number of distinct points. After

\footnotetext{
${ }^{3}$ Five video attachments have been uploaded together with this submission. They show the evolution of the representation for the interaction of the agent with the 5 environments of Figure 5
}

20 repetitions, the number of points in the representation has increased, and the structural cues starts shaping with $C_{2}=0.4$. They are visually well preserved starting from $C_{2}=0.6$ and 50 repetitions. After the almost convergence of $C_{2}$ around $10^{4}$ repetitions, the internal representation is visually a quasiperfect reproduction of the working space which is confirmed by a $C_{2}$ close to 1 .

b) Blobs environment: In this second environment, the environmental states are composed of randomly generated background images with black and white blobs stretched in the bottom left/top right direction of the working space, as plot in figure 3(c). The blobs comes from a random noise generated using a procedurial Perlin's noise with anisotropic filtering (steerable gaussian filter) oriented at 45 degrees. The resultant noisy image is then thresholded to give black and white blobs. The agent sensations are either 1 or 0 depending on the color of the blob the sensor is looking at. After each repetition, a new black and white image is randomly sampled. This process is repeated until $n=10^{6}$ explorations of the motor exploration set.

Looking at the refinement criterion $C_{1}$ represented in Figure 4, the refinement is ten times quicker than for the straight lines environment. Indeed in this environment and after successive environmental states, the sensory invariants are more localized because they lie inside the intersection of the successive blobs. This makes far poses quickly refined. This higher speed of refinement can be directly linked to the notion of a "richer" environment relatively to the agent's sensorimotor capabilities. In Figure 6, the local similarity criterion $C_{2}$ converges this time to a value of $C_{2}=0.77$ indicating a distortion of small dissimilarities in $\mathbf{D}$ with respect to the evaluation metric $\mathbf{R}$. Indeed, the sensory invariants have a higher probability to occur for a pair of points aligned along the top left/bottom right direction because the blobs are stretched in this direction. Thus, these pairs of points have a lower dissimilarity and are considered closer than in the orthogonal direction. The projected internal representation in Figure 5 shows as expected a stretch in the direction of high sensory invariants variance. However, after convergence, the structural cues are visually well preserved indicated by a high $C_{2}=0.77$, but not as well as in the previous environment.

Until now, the environmental states were kept fixed during the exploration, which gave an intuition about the distortion 


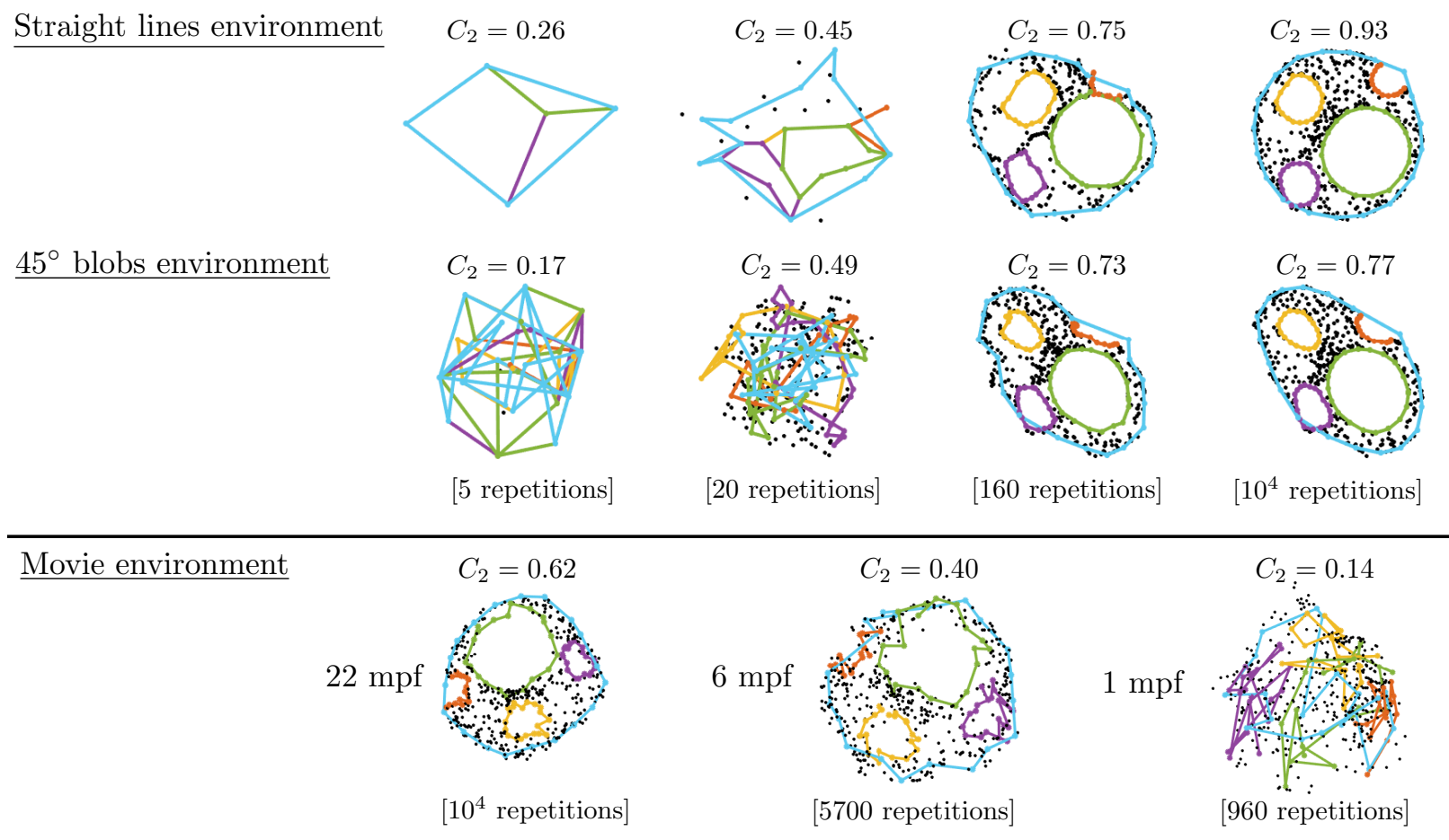

Fig. 5. At the top: visualization of criterion $C_{2}$ with the corresponding internal representations projected in $2 \mathrm{D}$ using Isomap with $K_{\max }$-NNs during the agent's life in the static environment scenario. At the bottom: final values of $C_{2}$ with the projected internal representations for the different dynamics of the movie environments.

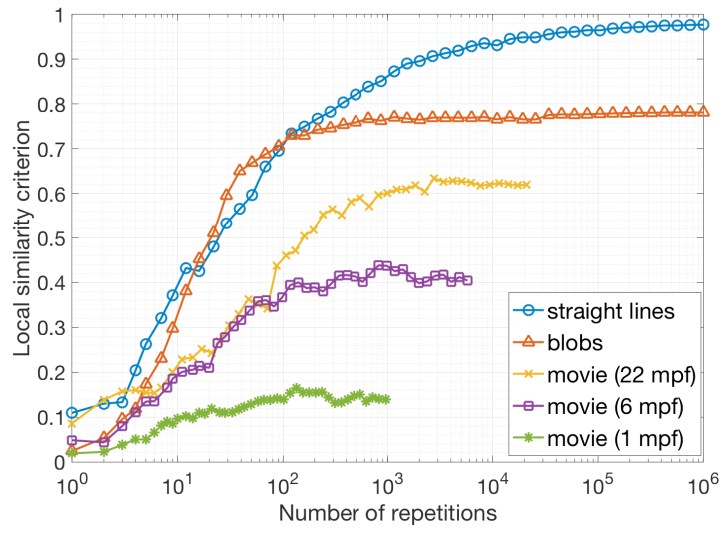

Fig. 6. Local similarity criterion evolution. (straight lines) converges to $C_{2}=$ 0.98 , (blobs) converges to $C_{2}=0.78$ and (movies $22 \mathrm{mpf}$ ) approximately converges to $C_{2}=0.62$, (movies $6 \mathrm{mpf}$ ) approximately converges to $C_{2}=$ 0.40 and (movies $1 \mathrm{mpf}$ ) converges towards $C_{2}=0.14$. The closer $C_{2}$ is to 1 the more accurate is the local structure of the internal representation.

in the representation as well as the notion of richness of the environment. Let's now consider more realistic dynamical environments.

2) Scenario 2, dynamical environment (Movie): In this last scenario, the environmental states are composed of cropped images of a black and white movie frames. One cropped image is shown in Figure 3(d). The agent hovers its sensor across the image; for that purpose, the agent has been centered inside the movie frame and scaled so that the diameter of its working space corresponds to 40 pixels in the image. In order to simulate a spatially continuous environment, the value given by its sensor is the linearly interpolated gray value at the $2 D$ sensor pose from adjacent pixels which is then quantified on a 0 to 15 range instead of the original 0 to 255 grayscale. Of course the agent's sensory inputs are uninterpreted: it cannot know that a value of 3 is closer to 4 than to 15 . Each sensory value is actually seen in this framework as a symbol. To simulate the dynamic of the environment, the image is refreshed with the next frame of the movie after a given number of movements. In the environments 3,4 and 5 the agent respectively moves at a speed of 22 motor configurations per frame (mpf), $6 \mathrm{mpf}$ and $1 \mathrm{mpf}$ : thus, a high mpf indicates a low dynamic of the environment. The movie file ${ }^{4}$ has been played 3 times, resulting in 479166 frames, which in turns corresponds to 21082 repetitions for environment 3,5749 repetitions for environment 4 and 960 repetitions for environment 5 .

Criterion $C_{1}$ plot in Figure 4 shows that the refinement is quicker in scenario 2 which indicates richer environments. The first reason comes from a higher number of sensory values: 16 gray-scale values instead of 0 and 1, making it less probable to have sensory invariants. The $1 \mathrm{mpf}$ environment reaches the finest refinement the quickest, indicating that quick refinement is also caused by a higher relative dynamic of sensory changes in the environment with respect to agent's movements. Moreover, Figure 6 exhibits that the $22 \mathrm{mpf}, 6 \mathrm{mpf}$ and $1 \mathrm{mpf}$ environments respectively converges to $C_{2}=0.62, C_{2}=0.4$ and $C_{2}=0.14$. In these dynamical environments, a pair of

\footnotetext{
${ }^{4}$ The selected movie is Phantom of the Opera (1943).
} 
close points in space but explored after a long time might have a distortion caused by the possible environment changes during the reaching time, thus the higher the dynamic the higher the distortion. At the bottom of Figure 5 are shown the final internal representations obtained after convergence of $C_{2}$. Visually the structural clues are well represented when dealing with a slow relative dynamic ( $22 \mathrm{mpf}$ and $6 \mathrm{mpf}$ movie environments). However, considering a high relative dynamic of $1 \mathrm{mpf}$, the agent's movements are too slow compared to sensory changes in the movie, which gives a scrambled representation close to a random permutation of points. In this last case, the internal representation is certainly not exploitable for any task defined in the working space because the agent cannot plan continuous trajectories.

\section{CONCLUSION}

This paper dealt with the question of how and why a totally naive (interpretation free) agent with access to its actuator states and sensors inputs can, through active exploration, build an internal representation of its sensors states in the physical world. It has been shown that, under the assumption of continuity on the statistics of sensory invariants in the physical space, this internal representation is topologically equivalent to a space called the quotient pose space. The quotient pose space represents the states of the agents that can't be distinguished by a sensory input (with probability 1 ). This space comes with a "natural topology" defined as the finest one that makes the movement of the sensors continuous. Then a formalization have been proposed to adapt the process of refinement to an experimental context with realistic environments. Proof of concept examples are shown in adequate environments: low dynamic relatively to the agent's movements, high probability of sensory invariants, but some limitations are presented when dealing with more challenging environments. A step forward would be to show a direct exploitation of the topological internal representation obtained after the refinement process in tasks such as path planning or obstacle avoidance. Moreover, the introduced concept of sensorimotor structure learning can be further applied as a background for the higher level considerations developed in interactive perception, but also to learn the structure of space based on compensatory actions, as a way to define the sensorimotor contingencies or a direction towards a sensorimotor definition of the notion of objects.

\section{REFERENCES}

[1] M. Charlet, E. Marcellini, and C. Gosselin, "Trajectory planning of projectile catching maneuvers for robotic manipulators," in International Design Engineering Technical Conferences and Computers and Information in Engineering Conference, 08 2018, p. V05BT07A014.

[2] Y. Zhao, X. Chai, F. Gao, and C. Qi, "Obstacle avoidance and motion planning scheme for a hexapod robot octopus-iii," Robotics and Autonomous Systems, vol. 103, pp. 199 - 212, 2018.

[3] D. Shaikh, L. Bodenhagen, and P. Manoonpong, "Concurrent intramodal learning enhances multisensory responses of symmetric crossmodal learning in robotic audio-visual tracking," Cognitive Systems Research, vol. 54 , pp. $138-153,2019$

[4] J. K. O'Regan and A. Noë, "A sensorimotor account of vision and visual consciousness." The Behavioral and brain sciences, vol. 24, no. 5, pp. 939-973; discussion 973-1031, Oct. 2001.

[5] J. K. O'Regan, "Solving the "real" mysteries of visual perception: The world as an outside memory," Canadian Journal of Psychology, vol. 46 no. 3, pp. 461-488, 1992.
[6] A. V. Terekhov and J. K. O’Regan, "Space as an invention of biological organisms," available at: arXiv:1308.2124v1 [cs.AI], August 2013.

[7] G. Le Clec'H, J. K. O'Regan, and B. Gas, "Acquisition of a space representation by a naive agent from sensorimotor invariance and proprioceptive compensation," International Journal of Advanced Robotic Systems, vol. 13, no. 6, 2016.

[8] H. Poincaré, "L'espace Et la Géométrie," Revue de Métaphysique Et de Morale, vol. 3, no. 6, pp. 631-646, 1895.

[9] __ "Science and hypothesis," London: Walter Scott Publishing, 1905.

[10] J. J. Gibson, The senses considered as perceptual systems. Boston: Houghton, 1966.

[11] P. Bach-y Rita, C. Collins, F. Saunders, B. White, and L. Scasdden, "Vision substitution by tactile image projection," Nature, vol. 221, no. 5184, pp. 963-964, mar 1969.

[12] J. J. Gibson, The ecological approach to visual perception. Boston: Houghton, 1979.

[13] A. Berthoz, Le sens du mouvement. Odile Jacob, 1997.

[14] A. Noë, Action in perception. The MIT Press, 2004.

[15] J. Bohg, K. Hausman, B. Sankaran, O. Brock, D. Kragic, S. Schaal, and G. S. Sukhatme, "Interactive perception: Leveraging action in perception and perception in action," IEEE Transactions on Robotics, vol. PP, no. 99 , pp. 1-19, 2017

[16] D. Philipona, J. K. O'Regan, and J.-P. Nadal, "Is there something out there?: Inferring space from sensorimotor dependencies," Neural Comput., vol. 15, no. 9, pp. 2029-2049, 2003.

[17] A. Laflaquière, J. K. O'Regan, S. Argentieri, B. Gas, and A. Terekhov, "Learning agents spatial configuration from sensorimotor invariants," Robotics and Autonomous Systems, vol. 71, pp. 49-59, September 2015.

[18] P. Demartines and J. Herault, "Curvilinear component analysis: a selforganizing neural network for nonlinear mapping of data sets." IEEE Transactions on Neural Networks, vol. 8, no. 1, pp. 148-54, jan 1997.

[19] V. Marcel, S. Argentieri, and B. Gas, "Building a sensorimotor representation of a naive agent's tactile space," IEEE Transactions on Cognitive and Developmental Systems, vol. 9, no. 2, pp. 141-152, June 2017.

[20] A. Laflaquiere, "Grounding the experience of a visual field through sensorimotor contingencies," Neurocomputing, 2017.

[21] V. Y. Roschin, A. Frolov, Y. Burnod, and M. Maier, "A neural network model for the acquisition of a spatial body scheme through sensorimotor interaction," Neural Computation, vol. 23, pp. 1821-1834, 2011.

[22] D. Philipona, J. K. O'Regan, J.-P. Nadal, and O. J.-M. Coenen, "Perception of the structure of the physical world using unknown sensors and effectors," Advances in Neural Information Processing Systems, vol. 15, 2004.

[23] A.A.Frolov, "Physiological basis of 3-D external space perception : approach of Henri Poincaré," in History of the neurosciences in France and Russia, hermann ed., 2011.

[24] L. Chen and A. Buja, "Local multidimensional scaling for nonlinear dimension reduction, graph drawing, and proximity analysis," Journal of the American Statistical Association, vol. 104, no. 485, pp. 209-219, 2009.

[25] J. A. Lee and M. Verleysen, "Scale-independent quality criteria for dimensionality reduction," Pattern Recognition Letters, vol. 31, pp. 2248 $-2257,2010$

[26] J. B. Tenenbaum, V. de Silva, and J. C. Langford, "A global geometric framework for nonlinear dimensionality reduction," Science, vol. 290, no. 5500, pp. 2319-2323, 2000.

[27] J. Bertrand, "Calcul des probabilités," p. 5, 1889.

[28] E. T. Jaynes, "The well-posed problem," Foundation of Physics, vol. 3, pp. 477-493, 1973. 